\title{
Preoperative screening cultures in the identification of staphylococci causing wound and valvular infections in cardiac surgery
}

\author{
E. J. Ridgway, A. P. R. Wilson and M. C. Kelsey \\ Department of Microbiology, Whittington Hospital, London, UK \\ Accepted for publication 21 September 1989
}

\begin{abstract}
Summary: Cultures of nasal or presternal swabs form part of the routine preoperative screening of patients on the cardiac surgical ward. During a trial of antibiotic prophylaxis in $\mathbf{3 1 4}$ patients, preoperative isolates of Staphylococcus aureus and coagulase-negative staphylococci were compared with strains associated with postoperative sternal wound breakdown (24 patients) and prosthetic valve endocarditis ( 3 patients). Morphology, antibiotic sensitivity pattern, plasmid analysis and phage typing were used to differentiate strains. In only three cases of wound infection and one of prosthetic valve endocarditis were pathogenic staphylococci not distinguishable from preoperative isolates. The collection of superficial swabs for this purpose before cardiac surgery is therefore unlikely to be cost effective.
\end{abstract}

Keyzords: Wound infection; heart surgery; Staphylococcus aureus.

\section{Introduction}

Preoperative screening of nasal or presternal swabs is commonly performed on cardiac surgical wards to identify patients carrying Staphylococcus aureus, particularly methicillin-resistant strains, that might later be responsible for sternal wound infection or prosthetic valve endocarditis (Keighley \& Burdon, 1979). In practice, preoperative isolation of Staphylococcus aureus has rarely, if ever, altered perioperative management and the commonest potential pathogen, Staphylococcus epidermidis, was often disregarded (Harris et al., 1984; Farrington et al., 1985, Wilson et al., 1988a). The cost of processing preoperative samples might be justified if the source of an operative infection could be identified as endogenous, and selective prophylaxis altered appropriately.

As part of a study of antibiotic prophylaxis (Wilson et al., 1988b), preoperative nasal or sternal swabs were collected from patients admitted

Correspondence to: Dr A. P. R. Wilson, Department of Clinical Microbiology, University College Hospital, Grafton Way, London WC1E 6AU, UK. 
for cardiac surgery. Preoperative isolates of staphylococci were compared with those isolated postoperatively from sternal wound infection or prosthetic valve endocarditis.

\section{Materials and Methods}

\section{Patients and preoperative cultures}

Cultures were obtained from patients who entered into a prospective randomized clinical trial to compare two prophylactic regimens for cardiac surgery: teicoplanin $(400 \mathrm{mg}$ iv with induction of anaesthesia and $200 \mathrm{mg}$ $24 \mathrm{~h}$ later), or flucloxacillin and tobramycin $(500 \mathrm{mg}$ flucloxacillin iv/po 6 hourly for $5 \mathrm{~d}$, tobramycin $80 \mathrm{mg} 8$ hourly iv for $3 \mathrm{~d}$ ) (Wilson et al., $1988 \mathrm{~b}$ ). Exclusion criteria were age under 18 years, pregnancy, a history of allergy to penicillin or vancomycin, serum creatinine over $150 \mu \mathrm{mol}^{-1}$, active infection or antibiotic treatment within the last 7 days.

Swabs were taken by the nursing staff from the anterior nares of all patients before and one week after operation. Cultures were made from the presternal skin immediately prior to operation. To increase the yield of bacteria, swabs were first moistened in sterile water. Postoperatively, swabs were taken from all discharging wounds and, in cases of endocarditis, cultures made from blood or the excised valve.

Wound infections were assessed by the ASEPSIS method (Wilson et al., 1986) in which a score is used to describe the extent and clinical significance of wound breakdown. A score less than 11 points indicates satisfactory healing of the wound, while minor, moderate and severe wound infections were defined by scores above 20,30 and 40 points, respectively. Early prosthetic-valve endocarditis was defined as the growth of an indistinguishable organism from two or more sets of blood cultures in the presence of fever, within 4 months of operation. The diagnosis was usually confirmed by the finding of vegetations at operation or post-mortem examination.

\section{Bacterial isolation and typing}

Any bacterial growth (one or two predominant colony types) after $48 \mathrm{~h}$ was identified by standard methods. When staphylococci were isolated in both preoperative and postoperative cultures, strains were differentiated by colonial morphology, pigmentation and haemolytic activity (Kloos \& Schleifer, 1975). Sensitivities to 14 antibiotics were performed by a modified Stokes method on Isosensitest agar with lysed blood (Oxoid) (i.e., methicillin, clindamycin, tobramycin, teicoplanin, novobiocin, penicillin, gentamicin, erythromycin, fusidic acid, trimethoprim, ciprofloxacin, vancomycin, rifampicin and tetracycline). Resistance to methicillin was tested by incubation at $30^{\circ} \mathrm{C}$. Strains were considered distinct if they differed by two or more unrelated resistance mechanisms.

All strains underwent plasmid analysis, by a method modified from 
Wilson, Totten \& Baldwin (1978). Briefly the strain was treated with lysostaphin followed by RNAse and protease. The plasmid DNA was precipitated with isopropanol and analysed by electrophoresis on a submerged agarose gel. Molecular weights were determined with reference to standards at $20,2.7,2.3$ and 1.8 megadaltons and strains were distinguished by one or more different plasmids.

Strains not differentiated by these methods were speciated by the API-Staph system and typed by bacteriophage at the Staphylococcal Reference Laboratory, Colindale. Strains were distinguishable if two or more strong differences were present in lysis by phages (Williams \& Rippon, 1952). Coagulase-negative staphylococci causing prosthetic valve endocarditis were also speciated and examined for slime production (Christensen et al., 1983).

\section{Results}

Of 419 patients who underwent cardiac surgery during the 15 months of the study, 314 completed the protocol. Coronary artery surgery was performed in 187 patients and valve replacement in 95 , the remainder having other intracardiac surgery.

Of the 314 patients postoperative sternal wound infections were recorded as follows: severe wound infection ( $>40$ points) in eight $(2.5 \%)$; moderate infection (31-40 points) in three cases (1\%); and minor infection (21-30 points) in $14(4.5 \%)$ others. Disturbance of healing (11-20 points) was noted in 24 patients $(7.6 \%)$. There were only two moderate and three minor leg-wound infections.

Preoperative nasal swabs were received from $280(89 \%)$ patients and swabs of the sternal skin from $133(42 \%)$ patients. Preoperative staphylococci were compared with those in the discharge from the sternal wound in 24 patients. In the three cases not shown in Table I, preoperative and postoperative strains were distinguished by coagulase, antibiogram and plasmids.

Wound sepsis due to Staphylococcus aureus

Prior to surgery, S. aureus was isolated from nasal swabs from $71(25 \%)$ of 280 patients and from $4(3 \%)$ of 133 swabs of the sternal skin. No methicillin-resistant strains were detected. $S$. aureus was present in both pre- and post-operative specimens in three patients, one with severe, one with minor infection, and one with disturbance of wound healing. Strains of $S$. aureus from the sternal wound of one patient and the leg wound of another were not distinguished from those in the preoperative nasal swab by any of the methods, including phage typing. Neither infection required antibiotics or drainage and the strains were sensitive to the prophylactic antibiotics used. 
E. J. Ridgway et al.

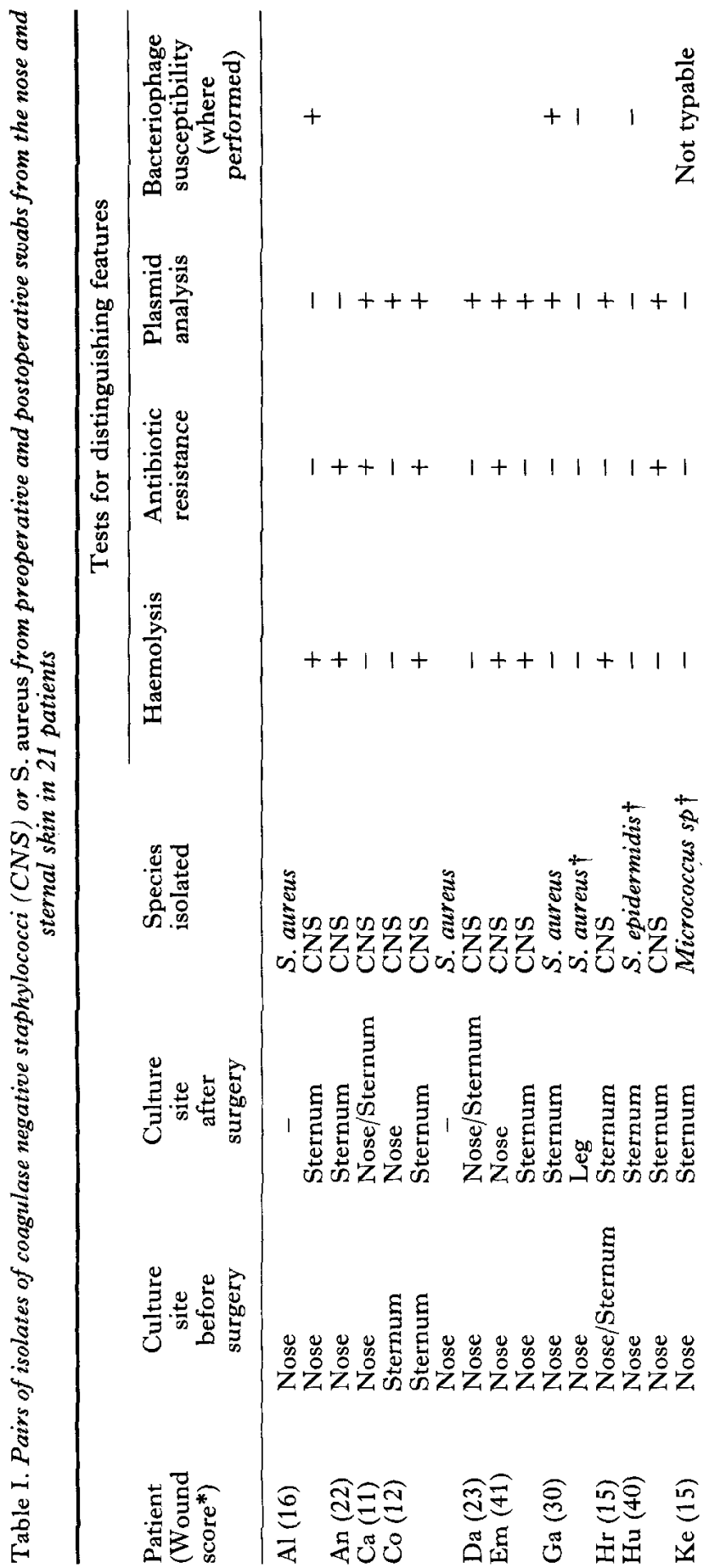




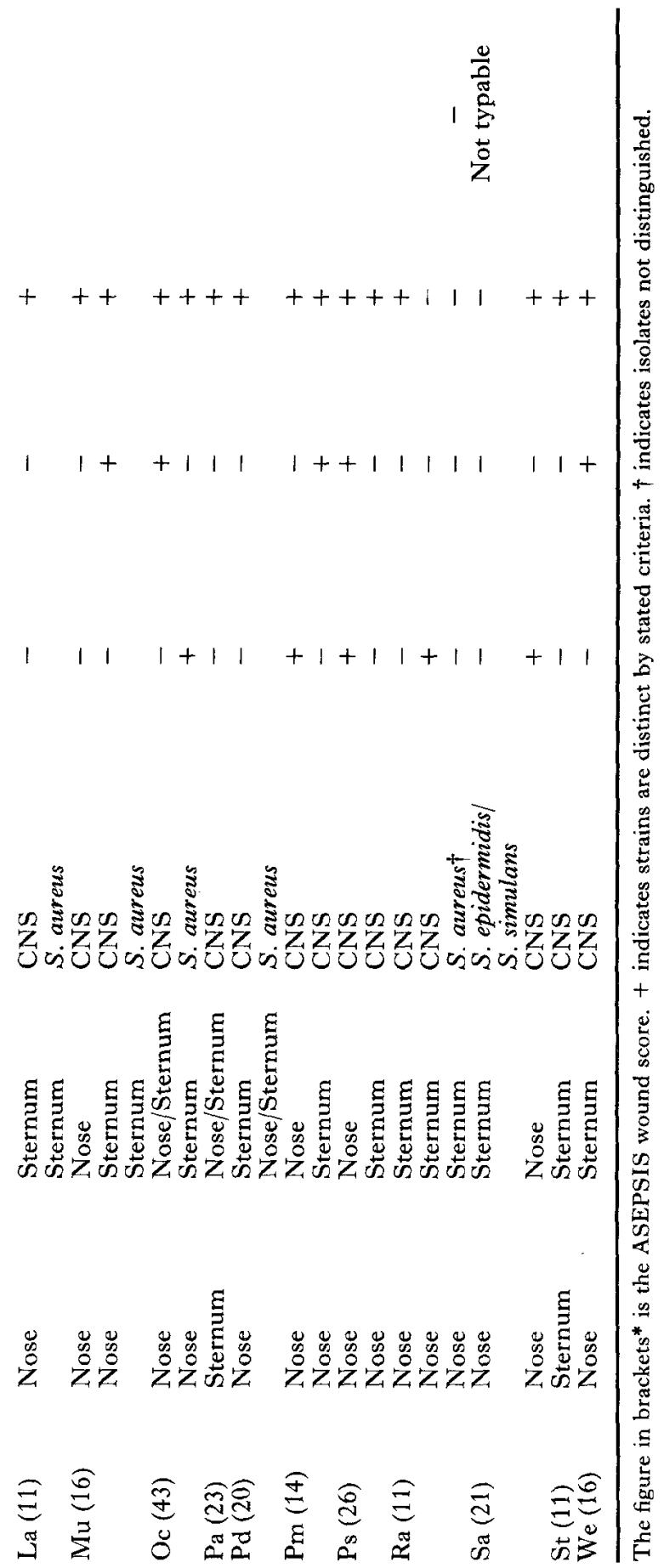




\section{Wound sepsis due to coagulase-negative staphylococci}

Coagulase-negative staphylococci were isolated from $205(73 \%)$ of 280 preoperative nasal swabs and from $89(67 \%)$ of 133 preoperative sternal swabs. The organisms were present in swabs before and after operation from 20 patients (two severe, one moderate and five minor infections and 12 with disturbance of healing), including two of the three patients described with paired isolates of $S$. aureus. Pairs of strains from five patients, not distinguished by other methods, were speciated and phage typed. Three pairs were of the same species, but only one pair had a similar bacteriophage profile (Staphylococcus epidermidis) and one pair was not typable (Micrococcus sp.). One wound required drainage under local anaesthesia and daily dressing at home but antibiotics were not needed in either case. Both organisms were sensitive to the prophylactic antibiotics.

\section{Early prosthetic-valve endocarditis}

Endocarditis developed in three of the 95 paticnts having valve replacement during the study (Table II). The preoperative sternal isolate and postoperative blood isolate could not be distinguished in one patient, but the organism, which was identified as Staphylococcus capitis, had no antibiotic resistances or plasmids and could not be phage typed. The patient, a 57-year-old man, was first noted to have heart block two months after aortic valve replacement but endocarditis with a para-aortic root abscess was not diagnosed until four months later. Treatment with parenteral penicillin and gentamicin resulted in a bacteriological cure but the patient died four days after replacement of the infected valve. Antibiotic prophylaxis for the original operation had been teicoplanin $(400 \mathrm{mg}$ and $200 \mathrm{mg} 24 \mathrm{~h}$ later iv), of which the MIC for the organism was $1 \mathrm{mg} \mathrm{l}^{-1}$.

\section{Discussion}

Preoperative nasal swabs predicted the subsequent infecting strain of staphylococcus in only three $(13 \%)$ of the 24 patients studied with sternal wounds that scored more than 10 points by the ASEPSIS method. Conventional criteria of wound infection, such as discharge of pus with crythema, would have identified only four of the 24 wounds as 'infected', the pathogen being similar to the preoperative strain in one case. As had been suspected, the estimated cost ( $£ 1300$ for 314 patients) of preoperative nasal swabs could not be justified for this purpose and no change in antibiotic prophylaxis was indicated.

Nasal swabs were collected from $89 \%$ of patients but the timing of sternal swabs on the day of operation presented practical difficulties. Consequently, results were not available in $48 \%$ of those with wound scores over 10 points. Those that were obtained did not predict subsequent wound infection or the causative pathogen. A sternal swab identified the source of the infecting organism in one case of prosthetic valve endocarditis, but serum 
Preoperative screening in cardiac surgery

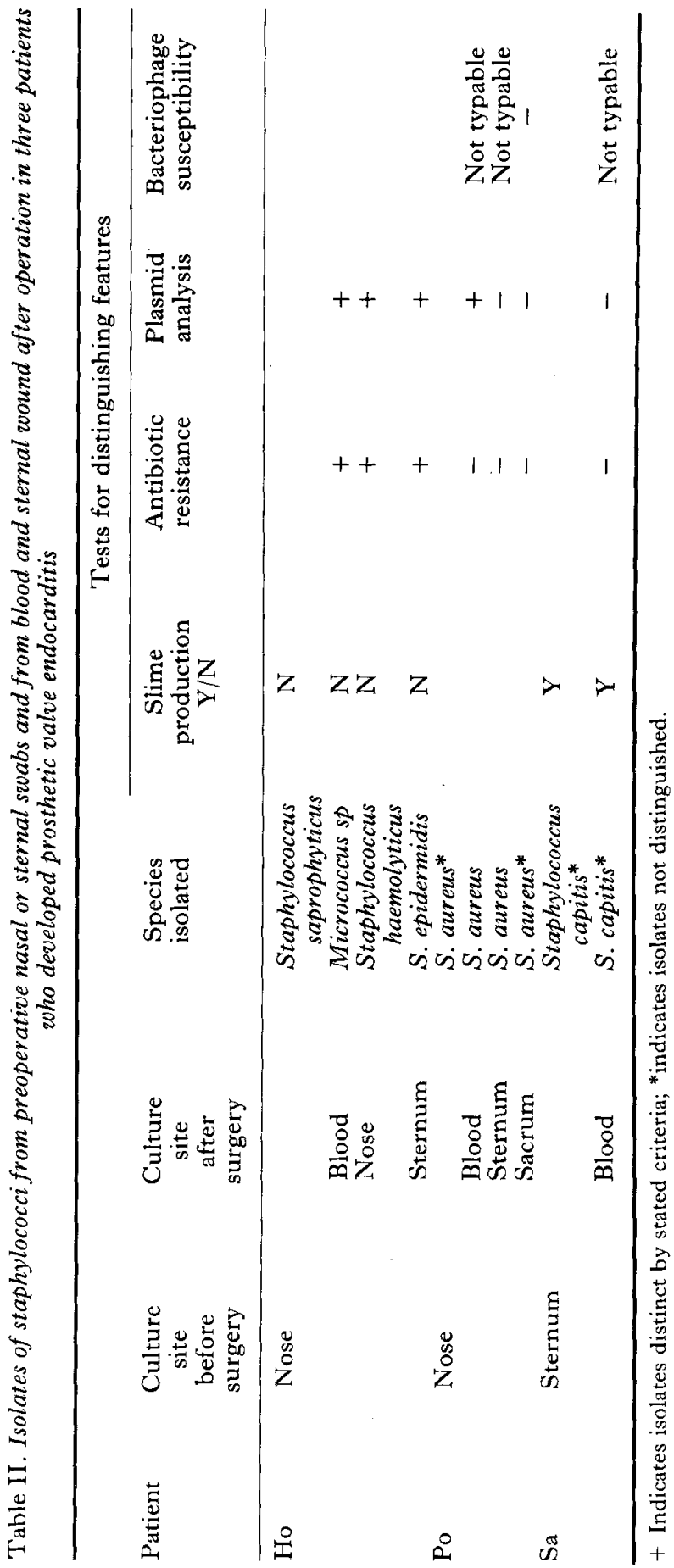


concentrations of the antibiotic used for prophylaxis would have been adequate to inhibit its growth (Wilson et al., 1988c).

Preoperative screening did not identify any carriers of methicillin-resistant strains of $S$. aureus, but this practice may be warranted to prevent the unsuspected introduction of a carrier into a regional centre. Earlier work has shown that S. epidermidis in pure growth is associated with wound sepsis of similar severity to that due to $S$. aureus (Wilson et al., 1988a). Screening for methicillin-resistant S. epidermidis might then be appropriate but would not have resulted in any change of management in this study.

Failure to isolate the infecting strain before operation does not necessarily indicate that the infections were exogenously acquired. Although nasal swabs were moistened to increase bacterial yield, fewer patients were found to carry $S$. aureus than might be expected from other studies (Williams, 1963). Repeated swabs collected by a trained investigator would have been necessary to establish the maximum ratc of carriage but that was neither practical nor could it be achieved routinely on a busy cardiothoracic unit.

Seeding of staphylococci from the patient's skin flora into the wound at operation is a common occurrence (Kluge et al., 1974). However, this study suggests that exogenous sources may be at least as important. Earlier work in this series of patients showed a single multiply-resistant strain of $S$. epidermidis to be associated with three severe wound infections (Wilson et al., 1988a). Houang et al. (1986) examined the possible origins of four index strains from sternal wound infections and concluded that the intensive-care unit team was the most likely source, in view of the frequency of carriage in that group. Preoperative samples had not been obtained. In a retrospective analysis, Marples (1980) found two strains of $S$. epidermidis to be responsible for nine infected wounds, and three infected valves. Similar strains with multiple antibiotic resistance were acquired by patients in the intensive care unit, a finding confirmed by Hansen (1982).

In this investigation, preoperative nasal and sternal isolates of staphylococci were collected prospectively and compared with those causing subsequent wound infection and endocarditis. This is rarely practicable in clean surgery and was only achieved in the case of this study as part of a major clinical trial. However, screening does not appear to be cost effective as a predictor of wound infection or as an aid to prophylaxis. It remains to be seen whether a more intensive study with repeated sampling from several sites preoperatively could determine the rate of endogenous infection.

We thank Mr T. Treasure and Mr M. F. Sturridge of the Department of Cardiothoracic Surgery of the Middlesex Hospital and Dr R. N. Grüneberg and the staff of the Department of Microbiology of University College Hospital for their help with the collection and initial processing of the samples. Thanks are also due to Dr R. R. Marples of the Staphylococcal Reference Laboratory, Colindale for phage typing of the isolates. 


\section{References}

Christensen, G. D., Parisi, J. T., Bisno, A. L., Simpson, W. A. \& Beachey, E. H. (1983). Characterization of clinically significant strains of coagulase-negative staphylococci. Fournal of Clinical Microbiology 18, 258-269.

Farrington, M., Webster, M., Fenn, A. \& Phillips, I. (1985). Study of cardiothoracic wound infection at St 'Thomas' I Iospital. British Yournal of Surgery 72, 759-762.

Hansen, B. G. (1982). The occurrence of Staphylococcus epidermidis in a department of thoracic and cardiovascular surgery. Scandinavian fournal of Thoracic and Cardiovascular Surgery 16, 269-274.

Harris, R. L., Wilson, W. R. \& Williams, T. W. (1984). Infections associated with prosthetic valves. In: Infections associated with prosthetic devices, (Sugarman, B. \& Young, E. J., Eds), pp. 89-112, Boca Raton, FL, USA: CRC Press Inc.

Houang, E. T., Marples, R. R., Weir, I., Mourant, A. J., de Saxe, M., Singleton, B. (1986). Problems in the investigation of an apparent outbreak of coagulase-negative staphylococcal septicaemia following cardiac surgery. Fournal of Hospital Infection 8 , 224-232.

Keighley, M. R. B. \& Burdon, D. W. (1979). Cardiovascular surgery. In: Antimicrobial prophylaxis in surgery, Chapter 8, p.136, Tunbridge Wells, UK: Pitman Medical Publishing Co.

Kloos, W. E. \& Schleifer, K. H. (1975). Isolation and characterisation of staphylococci from human skin. II Descriptions of four new species: Staphylococcus warneri, Staphylococcus capitis, Staphylococcus hominis and Staphylococcus simulans. International fournal of Systematic Bacteriology 25, 62-79.

Kluge, R. M., Calia, F. M., MacLaughlin, J. S. \& Ilornick, R. B. (1974). Sources of contamination in open heart surgery. Fournal of the American Medical Association 230, $1415-1418$.

Marples, R. R. (1980). Coagulase-negative staphylococci-their classification and problems. Royal Society of Medicine International Congress Symposium 23, 57-64.

Williams, R. E. O. (1963). Healthy carriage of Staphylococcus aureus: its prevalence and importance. Bacterological Reviews 27, 56-71.

Williams, R. E. O. \& Rippon, J. E. (1953). Bacteriophage typing of Staphylococcus aureus. Fournal of Hygiene (Cambridge) 50, 320-353.

Wilson, A. P. R., Treasure, T., Sturridge, M. F. \& Grüneberg, R. N. (1986). A scoring method (ASEPSIS) for postoperative wound infections for use in clinical trials of antibiotic prophylaxis. Lancet i, 311-313.

Wilson, A. P. R., Grüneberg, R. N., Treasure, T. \& Sturridge, M. F. (1988a). Staphylococcus epidermidis as a cause of postoperative wound infection after cardiac surgery: assessment of pathogenicity by a wound scoring method. British Yournal of Surgery 75, 168-170.

Wilson, A. P. R., Treasure, T., Grüneberg, R. N., Sturridge, M. F. \& Ross, D. N. (1988b). Antibiotic prophylaxis in cardiac surgery: a prospective comparison of two dose regimens of tcicoplanin with a combination of flucloxacillin and tobramycin. Fournal of Antimicrobial Chemotherapy 21, 213-233.

Wilson, A. P. R., Taylor, B., Treasure, T., Grüneberg, R. N., Patton, K., Felmingham, D. \& Sturridge, M. F. (1988c). Antibiotic prophylaxis in cardiac surgery: serum and tissue levels of teicoplanin, flucloxacillin and tobramycin. Fournal of Antimicrobial Chemotherapy 21, 201-212.

Wilson, C. R., Totten, P. A. \& Baldwin, J. N. (1978). Rapid procedure for the detection of plasmids in Staphylocoscus epidermidis. Applied and Environmental Micrabiolngy 36, 368-374. 